A Publication of the Systems \& Psychosocial Advances Research Center (formerly the Center for Mental Health Services Research) A Massachusetts Department of Mental Health Research Center of Excellence

Volume 11

Issue 1

2014

\section{Intimate Partner Violence in the Deaf Community: 5 Things You Need to Know \& 5 Things You Can Do}

Melissa Anderson, $\mathrm{PhD}$
A t least 500,000 people are members of the U.S Deaf community - a culturally distinct group of people who share American Sign Language (ASL) as a primary language (Mitchell, Young, Bachleda, \& Karchmer, 2006). For members of this community, Deaf is a cultural identity - not a disability - and is indicated by the capitalization of the letter D.

The Deaf community is rarely a focus of research. Few studies have been conducted on Deaf individuals' health and wellness, including their mental health needs, substance use, and experiences of violence and trauma. Gaps are particularly evident in the shortage of literature on Deaf people and intimate partner violence (IPV), defined as physical, sexual, or psychological harm by a current or former intimate partner or spouse (CDC, 2013). This absence wrongly implies that IPV is a non-issue in the Deaf community, an assumption that has been disproved by recent empirical and clinical findings. This Issue Brief details important facts about IPV within the Deaf community and provides specific recommendations for providers about best practices for working with Deaf clients.

\section{THINGS YOU NEED TO KNOW}

\section{Rates of reported IPV are higher among Deaf women than hearing women.}

Although it is commonly reported that $25 \%$ of women in the general population experience domestic violence in their lifetime, estimates within the Deaf community are closer to 50\% (Anderson \& Leigh, 2011). Rates of psychological, physical, and sexual victimization among Deaf college students and community women are significantly higher compared to their hearing peers (Anderson \& Leigh, 2011; Barnett et al., 2011).

\section{IPV may look different in the Deaf community.}

Emotional abuse may include the perpetrator insulting the Deaf victim by calling her "hearing" or making fun of her ASL skills. Intimidation may manifest as the perpetrator signing very close to the victim's face when angry, or overuse of floor stomping and pounding to get the victim's attention. Social isolation may be imposed through fear of the perpetrator's checking behavior - checking the victim's pager, email, or videophone logs. Economic abuse can be seen in the perpetrator's control of the victim's Social Security Disability Income checks (DeafHope, 2006).

\section{Hearing privilege can be used against Deaf victims/survivors of IPV.}

A unique characteristic of hearing-Deaf relationships is the potential for the hearing partner to abuse hearing privilege, the system of advantage based on hearing ability. From her experience working with Deaf survivors of IPV, Julie Rems-Smario (2007) has compiled an extensive list of examples of this 
abuse of hearing privilege: A hearing perpetrator does not inform the Deaf victim when people try to call; excludes the victim from important conversations and financial decisions; leaves the victim out of social situations with other hearing people; talks negatively about the Deaf community or disallows access to Deaf culture; criticizes the victim's speech and English skills; and manipulates police officers when they are called to the house.

\section{Deaf survivors may not label their experiences of IPV as “abuse”}

Many Deaf people lack information on what constitutes IPV and whether such violence is acceptable, due to a number of factors: limited family communication, reductions in incidental learning, lack of health education programs provided in ASL, etc. A recent study on IPV among Deaf female college students found that more than 50\% of Deaf survivors did not label their experiences of psychological aggression and physical assault as abuse, even when these experiences included severe harm (e.g., death threats, choking). An overwhelming majority of these survivors did not label partner-perpetrated sexual coercion as an abusive act (Anderson \& Kobek Pezzarossi, 2012). Similarly, Deaf rape survivors often struggle to define their experience at all, or label these experiences as miscommunication or bad sex (Elliott, 2012).

\section{Deaf survivors experience significant barriers to seeking help.}

Many agencies for hearing survivors of IPV do not provide communication accessibility for Deaf survivors (i.e., ASL interpreters, accessible hotlines, videophones). Survivors may avoid treatment due to valid concerns about confidentiality reduced anonymity within the close-knit Deaf community, fears about confidentiality among sign language interpreters, and unease about Deaf providers/ASL interpreters who belong to the same social circles as their clients (Barber, Wills, \& Smith, 2010). An additional barrier is Deaf people's substantial distrust of healthcare systems run largely by and for hearing people (Steinberg, Sullivan, \& Loew, 1998). A history of paternalism, bans on sign language, and eugenics is not easily forgotten.

\section{RECOMMENDATIONS FOR PROVIDERS}

\section{Tap Deaf clients' expertise on the unique communication needs of the deaf community.}

Build an effective alliance by meeting Deaf clients' unique language and communication needs, valuing Deaf culture, and respecting the client's survivorhood and Deafhood. Although providers' ASL fluency is typically the first priority of Deaf clients, a close second is working with a provider who has the "right attitude." For hearing providers who are not versed in Deaf culture or ASL, this means keeping an open mind, deferring to deaf clients' expertise in these areas, acknowledging the benefits one receives from a system of hearing privilege, and being willing to learn and adapt one's practice accordingly.

\section{Screen all clients for IPV - whether Deaf or hearing!}

Given the high rates of IPV within the Deaf community, IPV screening with Deaf clients is essential. Yet, Deaf individuals are frequently not screened for behavioral health problems. Screening for trauma may be even less likely, due to common misconceptions about romance and sexuality among Deaf individuals. For example, service providers often pay less attention to the sexual histories and sexual trauma experienced by women with disabilities, due to the incorrect belief that individuals with disabilities are not sexual beings (McRuer \& Mollow, 2012; Olkin, 1999).

\section{Use objective, behavior-driven language.}

When screening for IPV, use language that is objective and does not require the client's cognitive appraisal of their experiences. For example, say slap, punch, kick, curse, threaten, force and avoid using more clinical teams such as abuse, intimate partner violence, domestic violence, or coercion. These labels can be applied after sufficient psychoeducation has been provided.

\section{Address information gaps about IPV and health relationships.}

Investigate clients' knowledge of IPV and, if needed, provide education and resources. Additionally, direct clients to Deaffriendly resources such as DeafHope's Lavender Revolution or ASL videos created by SafePlace and Vera House.

\section{If needed, provide referrals to behavioral health treatment, preferably with a Deaf or ASL-fluent clinician.} If you feel that additional treatment for victims of IPV or trauma is needed, contact your state's commission for the Deaf and hard-of-hearing to obtain information about clinicians that provide specialized behavioral health services for Deaf clients.

*The image used in this brief depicts a model and not an actual victim or perpetrator of violence. 


\section{References}

Anderson, M. L., \& Kobek Pezzarossi, C. M. (2012). Is it abuse? Deaf female undergraduates' labeling of partner violence. Journal of Deaf Studies and Deaf Education, 17(2), 273-286.

Anderson, M. L., \& Leigh, I. W. (2011). Intimate partner violence against deaf female college students. Violence Against Women, 17(7), 822-834.

Barber, S., Wills, D., \& Smith, M. J. (2010). Deaf survivors of sexual assault. In I. W. Leigh (Ed.), Psychotherapy with deaf clients from diverse groups, Second Edition (pp. 320-340). Washington, DC: Gallaudet University Press.

Barnett, S., Klein, J. D., Pollard, R. Q., Samar, V., Schlehofer, D., Starr, M., Sutter, E., Yang, H., \& Pearson, T. A. (2011). Community participatory research with deaf sign language users to identify health inequities. American Journal of Public Health, 101(12), $2235-2238$.

CDC. (2013). Intimate partner violence. Retrieved from http://www.cdc.gov/violenceprevention/intimatepartnerviolence/

DeafHope. (2006). Deaf power and control wheel. Retrieved from http://www.deaf-hope.org/?page_id=6

Elliott, R. A. (2012). Disclosure of sexual assault among deaf female survivors. Unpublished thesis. Gallaudet University.

McRuer, R., \& Mollow, A. (Eds.). (2012). Sex and disability. Durham, NC: Duke University Press.

Mitchell, R., Young, T., Bachleda, B., \& Karchmer, M. (2006). How many people use ASL in the United States? Why estimates need updating. Sign Language Studies, 6, 306-335.

Olkin, R. (1999). What psychotherapists should know about disability. New York, NY: Guilford Press.

Rems-Smario, J. (2007). Domestic violence: We can’t ignore it anymore. NADmag, March/April 2007, 16-18. Retrieved from http://www.nad.org

Steinberg, A. G., Sullivan, V. J., \& Loew, R. C. (1998). Cultural and linguistic barriers to mental health service access: The Deaf consumer's perspective. American Journal of Psychiatry, 155(7), 982-984.

Intimate Partner Violence in the Deaf Community - M. Anderson 